Linguistique, littérature, didactique

151-152 | 2011

Anthropologies de la littérature

\title{
Le sacrifice de l'homme sauvage : un regard ethnocritique sur Jean-Luc persécuté de C. F. Ramuz
}

\section{Céline Cerny}

\section{(2) OpenEdition}

Journals

\section{Édition électronique}

URL : http://journals.openedition.org/pratiques/1856

DOI : 10.4000/pratiques. 1856

ISSN : 2425-2042

\section{Éditeur}

Centre de recherche sur les médiations (CREM)

\section{Édition imprimée}

Date de publication : 15 décembre 2011

Pagination : 257-271

\section{Référence électronique}

Céline Cerny, «Le sacrifice de l'homme sauvage : un regard ethnocritique sur Jean-Luc persécuté de $C$. F. Ramuz », Pratiques [En ligne], 151-152 | 2011, mis en ligne le 13 juin 2014, consulté le 19 avril 2019. URL : http://journals.openedition.org/pratiques/1856 ; DOI : 10.4000/pratiques.1856

(c) Tous droits réservés 


\section{Le sacrifice de l'homme sauvage : un regard ethnocritique sur Jean-Luc persécuté de C. F. Ramuz}

\section{Céline Cerny}

Université de Lausanne

\section{Circonstances de production}

En 1907, Charles Ferdinand Ramuz, jeune écrivain romand installé à Paris, est engagé par les éditions Payot à Lausanne pour réaliser un ouvrage sur le Valais en collaboration avec le peintre Edmond Bille. Au mois de juillet, il part donc à la découverte de cette région de montagnes qu'il ne connaît pas, et réside dans le village isolé de Chandolin où Edmond Bille possède un chalet. Jusqu'à 1'automne 1908, Ramuz multipliera les séjours en Valais, dans le village de Lens où demeure le peintre Albert Muret.

C'est au cours de cette période qu'il constitue un véritable corpus de travail - ébauches, notes de langue et de lieux, récits et légendes notamment - qu'il utilisera tout au long de sa carrière ${ }^{(1)}$. Tandis qu'il travaille à cet ouvrage de commande, Le Village dans la montagne (1908), portrait d'une communauté valaisanne au fil des saisons, Ramuz entreprend la rédaction de ce qui deviendra Jean-Luc persécuté ${ }^{(2)}$. Son journal témoigne à plusieurs reprises de l'impor-

(1) Sur l'utilisation récurrente du motif légendaire des âmes errantes des glaciers dans le corpus ramuzien, voir par exemple : Jérôme Berney, "Male mort", âmes errantes et purgatoire glaciaire. Autour du roman inachevé "Légende”", Etudes de lettres, « Dans 1'atelier de Ramuz », $\mathrm{N}^{\circ}$ 1-2, Lausanne, 2003, pp. 23-47. A ce propos, voir également : Claude Macherel, "La traversée du champ matrimonial : un exemple alpin », Etudes rurales, 1979, ${ }^{\circ}$ 73, pp. 9-40 ; «L'eau du glacier (Vallée du Lœtschental)», Etudes rurales, 1984, N 93-94, pp. 205-238 et «Un Purgatoire alpin (Lœtschental)», Le monde alpin et rhodanien. La haute montagne. Vision et représentation, Centre alpin et rhodanien d'ethnologie, Grenoble, 1988, pp. 87-112.

(2) L'histoire met en scène Jean-Luc et Christine, jeunes mariés, et leur bébé. Un soir Jean-Luc découvre que son épouse le trompe avec un amour de jeunesse. Il quitte alors le foyer, puis revient quelques mois plus tard. En coupant du bois, il se casse une jambe et cet accident rapproche Christine de son mari. Pourtant celle-ci recommence à voir son amant. Alors qu'elle attend un autre enfant, Jean-Luc la chasse définitivement et s'occupe désormais 
tance de ce projet, de plus grande valeur à ses yeux que le travail de collaboration effectué avec Edmond Bille, parce qu'il peut s'y exprimer en toute liberté et mettre en pratique ses recherches d'innovation esthétique. Ramuz travaille vite et le roman, qui d'après son auteur s'apparenterait plutôt à une longue nouvelle, sort de presse en novembre 1908, accompagné de deux autres histoires de la montagne ( «e Tout-Vieux »; " Alors il alla à la messe... »).

En vue d'une deuxième édition qui paraît en 1920, Ramuz reprend son texte et fait d'importantes modifications, supprimant par exemple le paragraphe qui clôt le roman. En 1930, les éditions Grasset publient une troisième version du texte, cette fois-ci légèrement différente. En 1940 enfin, dans le cadre de la publication de ses CEuvres complètes, l'écrivain se remet au travail et propose une dernière leçon de Jean-Luc persécuté( ${ }^{(3)}$. Le présent travail prend pour texte de référence l'édition originale de 1908, correspondant à la période où Ramuz, séjournant en Valais, est le plus proche de l'univers montagnard qu'il met en scène. C'est aussi durant les premières années de sa carrière que l'auteur vaudois, tout en poursuivant son œuvre solitaire entre la Suisse et Paris, participe activement au courant d'avant-garde artistique suisse romand. Membre fondateur de La Voile latine, il exprime à plusieurs reprises dans la revue la nécessité de l'indépendance de l'art et des artistes et la quête de nouvelles formes d'expression. Il souhaite créer un art dégagé des impératifs moraux, des contraintes politiques ou religieuses, un art capable de donner une image authentique de sa terre romande.

La représentation des paysages de montagne occupe une place importante dans cette recherche. Si Jean-Luc persécuté est le premier roman de Ramuz dont l'action se situe dans les montagnes du Valais, les nouvelles écrites entre 1902 et 1907 prennent souvent pour cadre les Alpes vaudoises ${ }^{(4)}$.

Depuis la fin du XIX ${ }^{\mathrm{e}}$ siècle, la tradition littéraire de Suisse romande s'est donnée des lieux et thèmes de prédilection, parmi lesquels la célébration d'un espace alpin pittoresque s'impose comme signe rassembleur d'identité nationale. Désireux de se distancer de cette peinture idyllique héritée de la tradition romantique, Ramuz présente le plus souvent des intrigues montagnardes tragiques. Il reproche aux écrivains qui l'ont précédé, de donner de la montagne une image figée et extérieure, et affirme la nécessité d'un rapport de familiarité et de proximité à son objet que viendra expliciter son essai Raison d'être (1914).

L'univers fictionnel de l'écrivain vaudois, à de rares exceptions, est celui d'un monde rural pré-industriel, souvent clos sur lui-même, privés de repères

seul de son fils. Jusqu'au jour où, durant Mardi gras, un masque jette le soupçon sur la paternité de Jean-Luc. Celui-ci se met à boire et délaisse son enfant qui se noie accidentellement dans un étang. A la suite de cette mort tragique, notre héros perd la raison et fait comme si le petit vivait encore. Christine de retour dans le village, Jean-Luc prétend que son fils a disparu. Pour le faire revenir, il va tuer sa femme et son nouveau bébé. Le roman s'achève sur la fuite de Jean-Luc dans la montagne et son suicide. Il se jette d'une falaise, persuadé que son fils perdu est à nouveau à ses côtés.

(3) L'édition des Romans de Ramuz dans la collection de la Pléiade (Gallimard) en 2005, dir. Doris Jakubec, propose, avec un appareil de variantes, les dernières versions de ces textes, soit celles mises au point par 1'auteur entre 1940 et 1941.

(4) Ces textes sont réunis dans C. F. Ramuz, Nouvelles et morceaux, 1, édités par C. Cerny et R. Mahrer, Euvres complètes, V, dir. D. Maggetti et R. Francillon, Genève, Slatkine, 2006. Désormais abrégé $O C$. 
spatiaux et temporels précis. Tout en choisissant cet univers terrien, Ramuz évite la description anecdotique des paysans, et cherche à dégager à travers la fiction, la simplicité essentielle de leur vie quotidienne. Il s'agit d'atteindre, à partir d'une observation locale et particulière, une vérité générale. Pour l'écrivain, la mise en œuvre de ce projet ne peut se faire sans sortir du cadre que le réalisme du XIX ${ }^{\mathrm{e}}$ siècle a donné au récit. Dans un même mouvement, Ramuz rejette l'esthétique naturaliste, condamnant les excès de l'explicatif, du documentaire, qui nuit selon lui à l'authenticité de l'œuvre. Cette prise de distance, l'auteur la met en scène en 1905 déjà dans "Sous la lune», nouvelle dialoguée où un écrivain, soucieux de s'émanciper des formes de la fiction réaliste, confie à son ami que « le roman doit être un poème » ${ }^{(5)}$.

Le cadre rustique et le travail sur la langue, qui tend à donner au texte le ton et le rythme du parler paysan - celui-là même des personnages -, favorise cependant une lecture restrictive de l'œuvre. En effet, une partie de la critique et du public romands, autant que le lectorat français, rangeront le plus souvent Ramuz parmi les écrivains régionalistes qui rencontrent en France, au début du XX ${ }^{\mathrm{e}}$ siècle, un important succès. Bien que profitant de cet engouement, Ramuz cherchera très vite à se dégager de cette image de poète du lieu et dira en 1914 :

On parle beaucoup ces temps-ci, de « régionalisme» : nous n'avons rien de commun avec ces amateurs de "folklore ». [...] Il ne faut pas que rien, dans ce que nous ferons, s'adresse à la curiosité du lecteur. ${ }^{(6)}$

\section{Le choix ethnocritique}

En situant Jean-Luc persécuté dans un décor alpin où évoluent personnages paysans et artisans dont la vie repose sur une économie de subsistance, dictée par le rythme des saisons, Ramuz favorise pourtant la présence de traits de culture populaire, voire de coutumes tenues pour traditionnelles. La critique ramuzienne s'est généralement contentée de relever la prédilection de Ramuz pour cet univers au mode de vie réputé ancestral, sans creuser plus avant. Les lectures de l'œuvre se sont plutôt arrêtées sur les motifs bibliques et la dimension christique du personnage de Jean-Luc, se contentant de décrire la couche symbolique la plus visible.

S'en tenir à cette interprétation sans prendre en considération « les tensions et les interactions entre traits culturels ${ }^{(7)}$, qui structurent la fiction et son réseau de signifiance, nous semble insuffisant pour mettre à jour la complexité du héros et les enjeux symboliques du roman. Une approche ethnocritique du texte qui « s'intéresse fondamentalement à la polyphonie culturelle » ${ }^{(8)}$ permet d'interroger ce matériau que Ramuz s'est réapproprié. De quelle manière apparaît le subs-

(5) «Sous la lune», Nouvelles et morceaux, 1, OC, V, 2006, p. 187.

(6) C. F. Ramuz, Raison d'être, voir Essais, 1, édités par R. Freudiger, OC, XV, 2009, p. 35.

(7) Présentation de l'orientation ethnocritique sur le site internet qui lui est consacré : www.ethnocritique.com

(8) Marie Scarpa, «Pour une lecture ethnocritique de la littérature », in Littérature et sciences humaines, textes réunis par A. Boissinot et al., Université de Cergy-Pontoise, 2001, p. 286. Voir également, l'ouvrage fondateur de ce courant critique : Jean-Marie Privat, Bovary Charivari, essai d'ethno-critique, Paris, CNRS éditions, 2002 [1994]. 
trat ethnographique dans la fiction ramuzienne et quel rôle joue-t-il à l'intérieur de l'économie générale de l'œuvre ? Dans quelle mesure Ramuz en tire-t-il parti sans pour autant céder à la tentation pittoresque ?

En considérant, avec Roger Chartier, que la culture est faite « d'appropriations, de réemplois, de détournements » ${ }^{(9)}$, nous aimerions montrer, comme le suggère Pierre Bourdieu, « tout ce que le récit doit à la réinterprétation que son auteur fait subir aux éléments primaires » : et comment ceux-ci « reçoivent un nouveau sens de leur insertion dans le système de relations constitutif de l'œuvre $[\ldots] .{ }^{(10)}$

\section{Jean-Luc et Christine : un couple hors-normes}

L'univers montagnard décrit par l'auteur renforce l'isolement des protagonistes et l'intrigue se déroule dans un microcosme, à l'intérieur d'une communauté restreinte où les impératifs économiques d'une production en autarcie ne dictent pas seulement le déroulement des activités quotidiennes, mais également les unions matrimoniales et la morale sexuelle.

En effet, comme le montre Martine Segalen, « le désir profond de contrôler l'avenir de l'exploitation règle la stratégie économique familiale qui vise à créer des alliances assurant l'accession à la propriété du sol, ou développant sa surface, ou encore limitant son morcellement. " ${ }^{(11)}$ Dans cette configuration, la conception moderne du couple conjugal, dont l'intimité prévaut sur les droits du groupe, n'existe pas : «[...] les relations au sein du ménage ne sont pas du domaine réservé aux époux, mais [...] elles sont socialisées et contrôlées par la communauté. ") ${ }^{(12)}$

Il serait donc anachronique de considérer l'histoire de Jean-Luc et Christine comme une passion vécue en vase clos, ignorant alors « la logique interne de la culture en question » ${ }^{(13)}$. Dans une société où la solidarité n'est pas un concept éthique mais bien une condition de survie, les relations affectives exclusives sont vues comme des menaces pesant sur l'ordre de la collectivité, qui considère la soumission de la femme à son mari comme faisant partie de la soumission des hommes à l'ordre commun.

Dès les premières lignes, le texte met en scène le jugement collectif du comportement individuel : parti à la recherche de Christine, Jean-Luc se fait interpeller par quelques hommes du village attablés devant l'auberge :

Mais, comme on l'invitait à venir boire, il refusa. Et les autres se mirent à rire : « Ça tient toujours! » « Est-ce permis ou non? » disait Jean-Luc. «Oh! c'est permis ! Il rit aussi, puis rentra vite. ${ }^{(14)}$

(9) Roger Chartier, «Histoire intellectuelle et histoire des mentalités. Trajectoires et questions ", Revue de synthèse, $3^{\mathrm{e}}$ trimestre, $\mathrm{n}^{\circ}$ 11-12, juillet-décembre 1983, Paris.

(10) Pierre Bourdieu, «Lecture, lecteurs, lettrés, littérature » (1981), Choses dites, Paris, Minuit, 1987, p. 141.

(11) Martine Segalen, Mari et femme dans la société paysanne, Paris, Flammarion, 1980, p. 19.

(12) Ibid., p. 30.

(13) Jean-Marie Privat, op. cit., p. 10.

(14) C. F. Ramuz, Jean-Luc persécuté, voir Romans, 1, édités par F. Ménand-Doumazane, R. Francillon et C. Cerny, OC, XIX, 2011, p. 400. 
En se moquant de cet attachement amoureux « qui tient toujours » et de l'attitude de Jean-Luc qui préfère la maison à l' auberge, les autres hommes mettent en évidence un comportement inhabituel, car « quiconque cherche à se singulariser introduit un trouble dans la bonne marche de la vie sociale. $\gg{ }^{(15)}$ En d'autres termes, en refusant de participer à ce moment de sociabilités masculines, Jean-Luc manifeste ici une incapacité à soustraire ses sentiments individuels au profit des conventions communautaires, dans un monde où " [1] a manifestation ou 1'expression [...] d'opinions personnel[1e]s ne sont pas encouragés par le code de valeurs et de normes. ")

Christine, elle aussi en transgression, enfreint les codes de la morale conjugale afin de cultiver son plaisir individuel, comme nous le montre ce passage où elle rappelle à Jean-Luc les circonstances de leur union :

Ecoute, dit-elle, il faudrait s'entendre. Tu te souviens pourtant, le jour du Patron, quand tu m'as demandé si je voulais bien, quand tu disais que tu m'aimais, je t'ai répondu : "Moi j'aime mieux Augustin, et il m'a demandée aussi, mais son père ne veut pas, parce que je suis trop pauvre, et moi j'en ai assez d'être servante chez les autres, je voudrais bien me marier, alors fiançons-nous, si tu veux ; seulement si Augustin veut m'embrasser, je me laisserai embrasser. » Est-ce vrai que je t'ai dit ça ? ${ }^{(17)}$

Tandis que Jean-Luc se trouve mieux au logis, Christine ne cesse de quitter le foyer. Or, dans le ménage rural traditionnel, les historiens et folkloristes s'accordent à dire que la maison est le lieu dévolu à la femme; son identité sociale y est cantonnée. A l'inverse, les tâches et le rôle du mari se situent à l'extérieur, dans les champs ou l'étable par exemple. Les attitudes allant à l'encontre de cette règle coutumière sont vues comme une transgression de l'ordre communautaire : d'une façon ou d'une autre la règle patriarcale qui induit la soumission et la passivité de la femme est mise à mal.

A plusieurs reprises, le récit met en scène le comportement "féminin 》 de Jean-Luc. Lorsque Christine quitte définitivement la maison par exemple, c'est lui qui prend en charge les tâches domestiques - refusant généralement l'aide de ses voisines -, se comparant à son épouse : c'est le monde à l'envers des proverbes et de l'imagerie traditionnelle.

Il rentra chez lui, fit bouillir de l'eau et se mit à laver les tasses et les assiettes. Puis il balaya la cuisine et, quand elle fut en ordre, comme midi venait, il fit cuire la soupe ; et il la donna à manger au petit, tendant maladroitement la grosse cuillère.

Puis posa ses mains devant lui, et il se disait: «Elles sont trop lourdes. Elle, elle les avait plus petites et légères ; seulement c'est des mains qui ont connu le mal.» Et il reprenait : «Ça, c'est les miennes. » Et il redressa la tête. «On s'en servi ra. ${ }^{(18)}$

(15) Henri Mendras, Sociétés paysannes, éléments pour une théorie de la paysannerie, Paris, Armand Colin, 1976, p. 80.

(16) Henri Mendras, La Fin des paysans. Changements et innovations dans les sociétés rurales françaises, Paris, Armand Colin, 1967, p. 15.

(17) Jean-Luc persécuté, op. cit., pp. 405-406.

(18) Jean-Luc persécuté, op. cit., p. 436. 
Cette «féminisation » du personnage est explicitée dans un plan détaillé du roman, précédant les phases rédactionnelles. Ramuz, parfaitement conscient de la portée symbolique d'une inversion des sexes, écrit à propos de Jean-Luc : « Il se met à faire le ménage comme une femme ${ }^{(19)}$.

Car si Jean-Luc agit « comme une femme », Christine, quant à elle, adopte le rôle traditionnel de l'homme en dictant les règles au sein du couple. Elle n'hésite pas d'ailleurs à manipuler son mari : pensons à la scène de colin-maillard, durant laquelle Jean-Luc, les yeux bandés, cherche en vain son épouse qui en profite pour embrasser son amant. Nous y reviendrons. Cette masculinisation est présente à différents moments du texte : lors de la mort de son père, c'est elle qui hérite des biens familiaux et vient s'installer dans sa chambre, se couchant dans « le lit du vieux », où « il y avait encore le creux. » ${ }^{(20)}$ La mise à mort de Christine aura lieu précisément lorsqu'elle travaille seule aux champs. Nous sommes bien en présence d'un couple aux rôles renversés ${ }^{(21)}$.

Dans la panoplie des symboles d'inversion, symptomatiques d'un désordre social, la permutation des sexes occupe une place importante. Au moment du carnaval par exemple, période du monde à l'envers autorisé, la pratique rituelle du changement de sexe par le déguisement rappelle que ce retournement ne peut être toléré par la communauté en-dehors d'un cadre temporel défini. La société traditionnelle considère effectivement le carnaval comme une période de désordre ponctuel, ritualisé et collectif.

Le comportement adultérin de Christine renforce cette situation de retournement, vécue comme une menace par le groupe, car mettant à mal l'ordre patriarcal où l'homme est chargé des avances. Ainsi, par la faute de Jean-Luc et Christine, s' « instaure l'avènement du monde à l'envers » ${ }^{(22)}$ permanent. Ou pour reprendre Suzanne Chappaz: «c'est en empié[tant] sur le territoire de l'autre sexe que le monde se retrouve cul par dessus tête. ${ }^{(23)}$

Cette formule n'est pas choisie par hasard car, ainsi que le rappellent Claude Gaignebet et Odile Ricoux, le cycle de carnaval est aussi, dans l'usage traditionnel, l'occasion de pratiquer la langue verte (de vertere : retourner), « où s'intervertissent lettres ou syllabes et où on détourne le sens des mots » ${ }^{(24)}$. Familier des sociétés d'étudiants où la pratique du calembour est fréquente, Ramuz ne de-

(19) «Jean-Luc Boitrun et tous les malheurs », 27 janvier 1908. Le dossier génétique de JeanLuc persécuté appartient au fonds privé C. F. Ramuz. Les titres des brouillons et ébauches cités sont donnés entre guillemets. Le patronyme Boitrun est remplacé par Robille dans la version définitive. Notons la proximité phonique entre ce nom Boitrun et les caractéristiques du personnage qu'il désigne. Jean-Luc étant tour à tour celui qui boite et celui qui boit trop...

(20) Jean-Luc persécuté, op. cit., p. 458.

(21) A propos des attentes sociales liées au genre et les questions d'ordre sexuel traditionnel, voir : Céline Cerny et Jérôme Meizoz, «L'écrivain et sa jeune fille en fleur. Ethnocritique d'une nouvelle de C. F. Ramuz, « Rose» (1905)», in Horizons ethnocritiques, sous la dir. de Jean-Marie Privat et Marie Scarpa, Presses universitaires de Nancy, 2010, pp. 33-46.

(22) Martine Segalen, op. cit., p. 41.

(23) Suzanne Chappaz, "Le cocu, la mégère et le "puto" ", in Les Femmes, sous la dir. de Jacques Hainard et Roland Kehr, MEN, Neuchâtel, 1992, p. 85.

(24) Claude Gaignebet et Odile Ricoux, «Les Pères de l'Eglise contre les fêtes païennes. Fous du Christ et Anes saturniens ", in Carnavals et mascarades, dir. Pier Giovanni d'Ayala et Martine Boiteux, Paris, Bordas, 1988, p. 47. 
vait pas ignorer ce que l'inversion obscène pouvait faire au prénom de son hé$\operatorname{ros}{ }^{(25)}$

On trouve dans la société traditionnelle européenne une coutume universellement répandue, chargée de combattre symboliquement les comportements horsnormes dont l'inversion des sexes et l'adultère féminin sont parmi les plus courants : le charivari ${ }^{(26)}$. Réservés aux couples allant à rebours des règles de conduite et menés par les jeunes gens de la communauté, les charivaris étaient faits en priorité lors de remariage de veufs et contre les maris battus ou trompés par leur femme. Ces manifestations de vindicte populaire, aujourd'hui disparues, étaient dirigées généralement contre les « individu[s] qui enfrei[gnent], d'une manière ou d'une autre, le code dominant de la morale sexuelle ou conjugale traditionnelle. » ${ }^{(27)}$ En tant que châtiment symbolique, « expression d'une contestation ou manifestation d'une "soupape de sûreté" " ${ }^{(28)}$, ils ont pour but : « de combattre un désordre par un acte de désordre, mais un désordre codé et symbolique, pour un ordre normalisé et réel. » ${ }^{(29)}$

Van Gennep décrit ainsi de nombreux rituels punitifs, dont la célèbre promenade assis à l'envers sur l'âne, réservés aux cocus; les cortèges où se mêlent vacarmes assourdissants, insultes et moquerie ; les mascarades où l'on déguise un mannequin en mari trompé ou encore la fête des cornards, assimilée à la traditionnelle Fête des Fous ${ }^{(30)}$.

Ces quelques éléments de culture populaire ancienne permettent de mettre à jour la dimension charivarique du couple formé par Jean-Luc et Christine, où chacun avance à rebours des conventions.

\section{Le charivari du masque à Mardi gras}

Van Gennep rappelle également que les charivaris aux maris battus ou cocus se concentraient souvent sur un seul jour de l'année, à Mardi gras ${ }^{(31)}$. Plusieurs auteurs attestent de ce genre de pratique dans les campagnes suisses ${ }^{(32)}$. Quant au carnaval, où le déguisement est de règle ${ }^{(33)}$, il occupe une place importante dans la société valaisanne traditionnelle de confession catholique. Parmi les pra-

(25) Jean-Marie Privat m'a rappelé d'ailleurs que Jean est le prénom traditionnel des cocus.

(26) A ce propos, voir l'ouvrage de référence d'Arnold Van Gennep, Le Folkore français. Du berceau à la tombe. Cycles de Carnaval-Carême et de Pâques, Paris, Robert Laffont, [1943], 1998 et Le Charivari, actes de la table ronde du 25-27 avril 1977 par 1'EHESS et le CRNS, publiés par Jacques Le Goff et Jean-Claude Schmitt, EHESS, Paris, Mouton Editeur, Paris, La Haye, New-York. Nous renvoyons également à Bovary Charivari de JeanMarie Privat (op. cit.) où cette pratique est largement analysée.

(27) Jean-Marie Privat, op. cit., p. 49.

(28) Jacques le Goff et Jean-Claude Schmitt, op. cit., « Le rituel : macro-analyse » de Isac Chiva et Françoise Zonabend, p. 366.

(29) Ibid., p. 75.

(30) Arnold Van Gennep, op. cit., p. 529 et sq. et p. 900 et $s q$.

(31) Ibid., p. 900.

(32) Arnold Niederer, "Mentalités et sensibilités ", in Histoire et Civilisations des Alpes, II, dir. Paul Guichonnet, Toulouse, Privat, Lausanne, Payot, 1980, p. 100. Louis Junod, « Le charivari au pays de Vaud dans le premier tiers du XIX ${ }^{\mathrm{e}}$ siècle », Archives suisses des traditions populaires, 47, Bâle, 1951, pp. 114-129.

(33) D’après Arnold Van Gennep : " Dans les Hautes-Alpes, les déguisements ont été de règle dans toutes les communes jusque vers 1860-1880 [...]. », op. cit., p. 758. 
tiques les plus répandues, la horde des masques terrifiants sévit encore aujourd'hui dans certaines régions. Pensons par exemple aux célèbres Tschäggätä dans la vallée du Lötschental (dans la partie germanophone du Valais), ces magnifiques masques en bois peint, aujourd'hui pièces d'art populaire. Cette survivance témoigne d'une pratique de très longue date qui respecte toujours, à peu de choses près, le même canevas; les jeunes gens du village déguisés et désormais anonymes viennent, à l'aide d'instruments bruyants, poursuivre les jeunes filles, se moquer des passants, entrer dans les maisons pour y semer la zizanie, et parfois plus, barbouiller les gens de suie ou leur lancer de la cendre ${ }^{(34)}$. La présence des masques se concentre généralement sur les jours précédents le Mardi gras, parfois n'apparaissent que ce jour-là, suivant les lieux et les époques.

Suzanne Chappaz décrit le carnaval comme le moment où se manifeste le jugement populaire des jeunes hommes de la communauté :

C'est à carnaval que s'exerce le plus souvent leur rôle justicier ; farces, procès parodiques et charivaris punissent les individus qui ont transgressé les règles de la collectivité : étrangers convoitant les filles du village ou du quartier, veufs remariés, maris cocus ou battus, femmes adultères, couples stériles, filles-mères et célibataires endurcis deviennent l'objet de la risée. ${ }^{(35)}$

Or, une partie du chapitre VI se déroule un Mardi gras, jour de la sortie des masques. Cette scène, que le narrateur introduit ainsi : «Quand, de nouveau, tout fut détruit en une fois » ${ }^{(36)}$, se révèle être un moment important de l'intrigue, un basculement dans le destin de Jean-Luc. Tandis que les hommes déguisés s'amusent à courir après les filles et les enfants, un homme masqué s'approche du héros et, sur le ton de la moquerie rituelle, lui demande d'où vient son fils, sous-entendant que l'enfant n'est pas de lui.

Les éléments folkloriques empruntés par Ramuz sont ici nombreux. En premier lieu l'allusion au travestissement : « les garçons habillés en elles », qui rappelle la configuration du couple précédemment décrite. Puis, sont décrits d'autres éléments de déguisement traditionnel comme « la figure cachée ou encore frottée de suie » ${ }^{(37)}$, les faux ventres, les changements de démarche ou de voix. Plus loin, on retrouve la coutume des cendres qu' on lance " à la figure des filles ». Enfin, le moment charivarique où le masque vient de manière détournée, comme c'était l'usage, dénoncer le statut du mari cocu en jetant un doute sur sa paternité. Désignant l'enfant, il dit : « Où l'as-tu trouvé celui-là ? », « L'as-tu payé cher?»

Le dossier génétique du roman contient différents brouillons de ce passage qui donnent à voir la manière dont Ramuz utilise ce matériau folklorique, réinvestissant les symboles qui en découlent au profit de son intrigue.

(34) A propos des carnavals du Valais, voir : Suzanne Chappaz, Le Turc, le Fol et le Dragon. Figures du carnaval haut-valaisan, Neuchâtel, Editions de 1'Institut d'ethnologie, Paris, Editions de la Maison des sciences de 1'homme, 1995. Mentionnons également 1'ouvrage réalisé par le photographe Albert Nyfeler et l'écrivain Maurice Chappaz, Lötschental secret, Lausanne, Editions 24 Heures, 1975.

(35) Suzanne Chappaz, Le Turc, le Fol et le Dragon, op. cit., p. 101.

(36) Jean-Luc persécuté, op. cit. Cette citation et les trois suivantes, p. 438.

(37) Ibid., p. 439. 
N'oublions pas également que Ramuz, jeune citadin de culture protestante, n'est pas originaire du Valais. De plus, son journal nous apprend qu'il n'a sans doute jamais vu les masques de Mardi gras au moment où il écrit son texte. En effet, son unique séjour hivernal à Lens débute exactement le 4 mars 1908, soit le mercredi des cendres, lendemain de Mardi gras qui eût lieu cette année-là le 3 mars justement ${ }^{(38)}$. Nous savons aujourd'hui que Ramuz a fréquenté les écrits des folkloristes, en particulier les recueils de contes et légendes du Valais. Sa connaissance des traditions populaires et des modes de vie valaisans combine donc savoir livresque, observations personnelles et récits oraux glanés tout au long de ses séjours ${ }^{(39)}$.

Parmi les documents conservés, on trouve une version de cette scène, datée du 17 mars 1908. Appartenant vraisemblablement au manuscrit définitif, elle a été écartée par l'auteur et réécrite. En voici quelques extraits :

[...] Il y avait une douzaine de ces masques, qui avaient mis toutes sortes de vieux habits, et les garçons portaient des jupes avec des coussins par devant et des corsages de couleur, un avait une ombrelle rouge toute crevée, un autre un panier sur la tête avec/et un châle qui lui pendait sur la figure, et un jouant sur les orguettes, les autres allaient derrière en se donnant le bras, tandis que les filles par dessus leurs robes avaient des vestes de garçons et des chapeaux de soie, [...].

Et ils venaient/passaient ainsi, se moquant de ceux qui étaient assis le long de la rue, leur criant des plaisanteries, changeaient leurs voix pour ne pas être reconnus. $[\ldots]$

Et les vieux qui sont un peu en dehors de la vie regardaient sans rire, penchés en avant sur leurs hauts genoux, tandis qu' on s'amusait autour, les jeunes femmes et les filles riaient et les enfants qui se sauvaient en riant. Surtout à cause d'un qui venait d'arriver, un grand avec un sac de cuir pendu au bout d'une courroie et plein de cendres qu'il jetait à la poignée/par poignée à la figure des filles, et on les entendaient crier aussi, parmi les rires, qui se sauvaient aussi - et le grand leur courait après. Il avait sous une espèce de casquette une toile de sac serrée autour du cou et dessus étaient peints des yeux rouges avec un nez vert/un triangle vert qui était le nez et une grande bouche ouverte qui montait jusqu'aux oreilles.

Alors à un moment il vint près de Jean-Luc il s'était approché devant lui, se retournant devant lui les jambes écartées, il le regarda et montrant le petit lui dit avec une voix comme une voix de femme : ${ }^{(40)}$

Le brouillon s'achève ici. Cette version antérieure accorde une plus large part à la description des déguisements, produisant un effet de distance documentaire. Le lecteur n'est plus parti prenant d'une intrigue dont il comprend les enjeux, mais spectateur d'un tableau pittoresque s'insérant plus difficilement dans la

(38) C. F. Ramuz, Journal, 2, OC, II, 2005, p. 105. Ramuz fait deux séjours en Valais cette année-1à, du 4 mars au 16 mai, puis du 22 septembre au 20 octobre.

(39) Voir à ce propos : Jérôme Berney, «La Grande peur dans la montagne de C.F. Ramuz ou la naissance d'une légende », A contrario [revue interdisciplinaire de sciences sociales], vol. 4, $\mathrm{n}^{\circ} 1,2006$, Lausanne, Antipodes, pp. 53-70 et Céline Cerny, « Un regard ethnocritique sur l'œuvre de Ramuz : 1'exemple du légendaire », A contrario, vol. 4, n² 2, 2006, Lausanne, Antipodes, pp. 25-35.

(40) Fonds privé C. F. Ramuz, «Le jour du mardi gras, les bouffons... ». Pour des raisons de lisibilité, cette transcription du manuscrit ne donne pas à voir les segments biffés et/ou remplacés. Les leçons concurrentes sont conservées en apposition et séparées par une barre oblique. 
dramaturgie. Dans la rédaction définitive, la partie descriptive est réduite pour laisser la place à cette confrontation entre Jean-Luc et le masque.

Deuxièmement, le masque qui vient parler à Jean-Luc, et dont Ramuz détaille le grimage, n'est pas encore individualisé. Dans la version publiée, on reconnaît Anthime, le voisin ennemi qui n'est plus un figurant folklorique mais devient un personnage à part entière. Ramuz s'approprie ici le motif populaire de la visite des masques à Mardi gras pour renforcer la tension dramatique d'un moment charnière de son récit et mettre en avant le caractère marginal de son héros. On le constate en lisant le dossier génétique du roman, l'idée du doute qui s'insinue peu à peu dans l'esprit de Jean-Luc est présente, dans les brouillons, à différents moments de l'intrigue, pour trouver sa place définitive dans cette scène de carnaval. Réagissant à l'opposé des conventions où la victime accepte généralement les plaisanteries faites à ses dépens ${ }^{(41)}$, Jean-Luc quitte les lieux. Profondément ébranlé, il ne semble pas saisir la dimension charivarique de la remarque, témoignant une fois de plus de son inadéquation : il n'est plus seulement le mari cocu, ou celui qui se comporte de manière trop féminine, mais aussi désormais celui qui vit en dehors du temps traditionnel où le rituel est intimement lié au rythme calendaire.

\section{Du cocu au fou et jusqu'au reniement}

Dès le lendemain, premier jour de Carême et donc de tempérance, Jean-Luc commence à boire et délaisse son fils. Pendant que Christine, qui ne reçoit quant à elle aucun châtiment populaire, disparaît pratiquement du récit jusqu'à la mort de son père, Jean-Luc ne cesse de manifester son dérèglement, témoignant de son inadaptation.

La mort accidentelle de son fils qui survient tandis que Jean-Luc est à l'auberge, fait perdre à ce dernier toute notion de réalité. Dès le lendemain de l'enterrement, il vit comme si l'enfant était encore présent à ses côtés et consacre toute son énergie à cet être imaginaire. Du fou figuré, représentation carnavalesque du monde renversé, et que la tradition montre parfois en cocu cornu, Jean-Luc devient le fou permanent. Sa folie manifeste sa non-conformité à l'ordre social et se signale à plusieurs reprises par un fonctionnement à contretemps. Il serait trop long d'en faire une liste ici, soulignons simplement qu'une fois devenu fou, Jean-Luc porte «toute la semaine », ses « habits noirs du dimanche » ${ }^{(42)}$.

A plusieurs reprises, l'on voit également Jean-Luc négliger son bien. Quand il se met à boire, il commence par vendre l'une de ses vaches - bête extrêmement précieuse dans cette société montagnarde où domine l'élevage - et vend certains de ces prés. Après une soirée passée à l'auberge, il va jusqu'à brûler de l'argent.

Le retour imaginaire de l'enfant ne pousse cependant pas Jean-Luc à reprendre ses activités agricoles. Un jour de printemps, son cousin le surprend en train de cueillir les fleurs de son pommier et d'en faire « un bouquet pour le petit». Encore une fois, Jean-Luc agit à contretemps : en cueillant la fleur sans attendre le fruit, il va à l'encontre des règles élémentaires de la production agraire, malgré

(41) A ce propos, voir encore Arnold Van Gennep, op. cit., pp. 900-903.

(42) Jean-Luc persécuté, op. cit., p. 457. 
les remarques de Théodule : «Laisse-lui plutôt les fruits », et de sa femme : «Mon Dieu! est-ce possible ? Tout ça de moins, tout ça de perdu ! » ${ }^{(43)}$

Cette folie douce vécue par les habitants du village comme l'est l'innocence bête des idiots, devient effrayante et menaçante après le retour de Christine qui, d'après Jean-Luc, a provoqué la fuite de l'enfant imaginaire. Désormais obnubilé par cette disparition, il passe son temps entre les bois et la montagne alentour dans une recherche sans trêve qui le conduit, un dimanche après-midi, jusqu'au hameau de sa mère. Mais cette dernière, au lieu de l'accueillir, condamne sans appel sa conduite, allant jusqu'à remettre en question le lien qui les unit :

[...] et de qui es-tu ? ils disent que tu es de moi, eh bien je ne peux plus le croire, quand je te regarde je pense : «Est-ce que c'est mon fils ? 》Je te répète, mange et bois, et ensuite tu remonteras, parce que je ne te connais plus. ${ }^{(4)}$

Tout se passe comme si un doute général pesait sur la filiation de Jean-Luc, participant au rejet dont il va être l'objet ; est-il bien le père de son fils, est-il même le fils de sa mère?

Saturée d'éléments symboliques, cette scène du reniement offre de multiples lectures. Concentrons-nous cependant sur la matière folklorique enfouie à l'intérieur du texte et qu'il s'agit de mettre à jour.

Le « dialogue de sourds » entre le héros et sa mère trahit l'impossible intégration de ce dernier aux lois de la communauté, pour prononcer enfin sa condamnation définitive. Les paroles de Jean-Luc lui-même affirment d'ailleurs sa différence consciente. Persuadé d'être désormais le seul à voir ce que les autres ignorent, il dit à sa mère : "C'est que tu es aveugle ; tu entends, c'est moi qui voit clair. » Puis de manière redondante : «J'ai des oreilles, j'ai des yeux et puis des oreilles. » ${ }^{(45)}$ La communauté villageoise, à qui le récit donne la parole, ne cesse pourtant de rappeler son aveuglement amoureux : «C'est Christine qui a eu tort, non pas lui, il a beaucoup de qualités, au fond, seulement il ne voit pas clair. »" (46)

La scène de colin-maillard, au chapitre IV, est exemplaire à cet égard puisque c'est Christine elle-même qui bande les yeux de Jean-Luc et s'assure qu'il ne voit rien pour mieux embrasser Augustin. Rappelons que c'est sur l'invitation de Jean-Luc que l'amant se trouve alors dans la maison du couple pour y boire un verre. L'aveuglement du mari cocu est d'ailleurs un motif traditionnel bien connu et Van Gennep décrit notamment une mascarade de Mardi gras où un mannequin «bien encorné », représentant l'époux trompé refusant de payer la Justice des Fous, « est placé sur le chariot fatal avec un bâton à la main ou un mouchoir sur les yeux. » ${ }^{(47)}$

Les paroles de la mère, d'une grande violence, disent en réalité le jugement de la collectivité : ce n'est pas tellement la mort du petit qu'elle reproche à son fils mais d'avoir gâché le bien familial et rompu le cycle de production. Elle condamne également le choix de son épouse, celle qui lui a fait «pousser les cor-

(43) Ibid., p. 455.

(44) Ibid., p. 462.

(45) Ibid., p. 462.

(46) Ibid., p. 436.

(47) Arnold Van Gennep, op. cit., p. 905. 
nes " ${ }^{(48)}$. C'est l'unique mention explicite des attributs traditionnels du cocu dans tout le récit, introduisant un accessoire supplémentaire à l'identité symbolique de Jean-Luc le porteur de cornes.

L'opposition entre le groupe et l'individu isolé qu'est Jean-Luc se manifeste également à travers l'intervention des hommes du voisinage. Ces mêmes hommes qui, quelques lignes auparavant, proposaient au héros de se joindre à eux - configuration similaire à celle de l'ouverture du roman et même refus -, sont prêts à attacher Jean-Luc avec une corde pour le faire sortir. Menée par Baptiste le chasseur aux deux chiens, cette intervention musclée annonce en fait clairement la course finale dans la montagne où quatre villageois partent à la recherche du coupable.

\section{La chasse à l'homme sauvage}

En effet, dans la dernière partie du récit, les justiciers prennent bien vite des allures de chasseurs « piqués au jeu et excités par cette fuite ${ }^{(49)}$. Les voilà lancés à la poursuite de l'homme à l'envers, le cornu ou le fou, celui que Marie la voisine et amie s'est d'ailleurs mis à vousoyer et à traiter de sauvage ${ }^{(50)}$. L'image de deux petites filles qui, le lendemain du reniement, « traîn[ent] un bélier par les cornes $»{ }^{(51)}$ préfigure également la capture de la bête.

Parmi les pratiques charivariques des sociétés traditionnelles, la « chasse à l'homme sauvage ", représentant la victime expiatoire de la communauté, est présente sous différentes formes rituelles. La dernière course de Jean-Luc, qui s'achève dans la mort, peut être lue comme une version littéraire de cette chasse folklorique, en tant qu'ultime châtiment populaire infligé à celui qui cumule les comportements hors-norme. Jean-Marie Privat rappelle en effet que traditionnellement, le « mannequin de Carnaval [...], avant d'être livré aux flammes sous les regards curieux de la foule, [est] accusé de tous les crimes et de tous les scandales [...]. » ${ }^{(52)}$ C'est bien le même rôle sacrificiel que joue l'homme sauvage traqué, le mari encorné, le mannequin de Mardi gras ou encore le Roi des Fous.

Suzanne Chappaz donne une description détaillée du rôle critique dévolu aux jeunes gens de la communauté par l'intermédiaire d'une chasse similaire :

Il était fréquent, dans de nombreux villages haut-valaisans, qu'ils mettent en scène à l'époque de carnaval le jeu de l' " homme sauvage », prétexte pour épingler [...] les individus ayant porté atteinte aux bonnes mœurs. [...] l'homme sauvage, vêtu de peaux de bête ou d'une tunique constituée de mousse, de feuillage ou recouverte de coquilles d'escargot, boute le feu à la hutte d'un ermite après avoir accompli dans le village plusieurs méfaits. Les habitants [...] envoient des chasseurs à sa poursuite. Ceux-ci gagnent les versants voisins où l'homme sauvage s'est enfui, le capturent, l'enchaînent et l'amènent sur la place du village où siègent le juge et la cour. Le « procureur » déroule un long parchemin et entre-

(48) En tant que représentante de la parole communautaire, c'est elle aussi qui dit à Jean-Luc : «Ils avaient bien raison!» « Ceux qui disent que tu es fou !», p. 462.

(49) Jean-Luc persécuté, op. cit., p. 477.

(50) Ibid., p. 459.

(51) Ibid., p. 464.

(52) Jean-Marie Privat, Bovary Charivari, op. cit., p. 177. 
prend la lecture de l'acte d'accusation. Ce procès, qui attribue à l'homme sauvage les malheurs survenus pendant l'année écoulée, permet aux garçons d'exercer leur rôle justicier sous la forme d'allusions mordantes à l'adresse des individus accusés de transgresser les règles de la collectivité. L'homme sauvage tente de s'enfuir, en vain. Condamné à mort, il est abattu et s'écroule en faisant éclater dans sa chute une vessie de porc remplie de sang, dissimulée sous sa tunique. ${ }^{(53)}$

Comme l'homme sauvage, Jean-Luc est coupable de différents méfaits et s'enfuit dans la montagne après avoir mis le feu.

L'archétype de l'homme sauvage, représentant l'être hors civilisation, l'exclu, le sacrifié, et, par son sacrifice, celui qui restaure l'ordre du monde, peut être interprété de différentes façons. En décrivant les chasses à l'ours des Pyrénées, où des hommes déguisés et le visage barbouillé de noir sont poursuivis, rasés puis mis à mort, Van Gennep rappelle que :

La chasse rituelle, exécutée par des chasseurs sélectionnés, d'un animal sauvage, est représentatif, soit comme le prétend Violet Alford, de l'Hiver qui doit finir, soit, selon les théories de Mannhardt-Frazer, de l'Esprit de la Végétation, qui, comme dans les cultes d'Osiris en Egypte, d'Adonis en Phénicie, puis des cultures du même type en Indonésie, en Extrême-Orient, en pays slaves et germaniques, tour à tour meurt, puis, grâce à certains rites et à certaines incantations, ressuscite annuellement. ${ }^{(54)}$

En effet, en tant que symbole traditionnel du renouvellement annuel et du cycle saisonnier, le porteur de cornes, sur le modèle du cerf dont les bois tombent et repoussent chaque année ${ }^{(55)}$, est celui qui, par son sacrifice restaure l'ordre du temps. Sa mise à mort symbolique permet d'une part le passage calendaire et d'autre part, le retour à l'ordre social ${ }^{(56)}$.

L'acte propitiatoire renvoie également au sacrifice du bouc des fêtes de Dionysos, dieu des masques et inventeur du théâtre. N'est-ce pas d'ailleurs le bouc qui a donné son nom à la tragédie, mot dérivé dans lequel on reconnaît les termes grecs tragos «bouc», et odê «chant » ${ }^{(57)}$ ? Cette tragédie dont l'essence même est de raconter indéfiniment l'affrontement d'Eros et Thanatos, l'amour conduisant à la mort, et dont l'histoire de Jean-Luc et Christine serait une nouvelle représentation.

Souvenons-nous en effet que Ramuz lui-même - et ce n'est pas la seule fois -, fait mention du genre pour décrire la dimension universelle de ses personnages :

Mes personnages ont donc été des paysans. Mais j'ajoute tout de suite que, s'ils ont été des paysans, ce n'est nullement que j'aie été préoccupé de peindre les mœurs d'une classe $[\ldots]$; c'est parce que, attachés à une nature grande et reliés à elle par une antique fidélité, ils me paraissent grâce à elles susceptibles d'une certaine grandeur, disons poétique, [...] qu'ils me paraissaient d'autre part infini-

(53) Suzanne Chappaz, Le Turc, le Fol et le Dragon, op. cit., p. 104.

(54) Arnold Van Gennep, op. cit., p. 772.

(55) Voir à ce propos, Claude Gaignebet et Odile Ricoux, op. cit., p. 47 et sq.

(56) Suzanne Chappaz, Le Turc, le Fol et le Dragon, op. cit., p. 262.

(57) A ce propos, voir Jean Chevalier et Alain Gheerbrant, Dictionnaire des symboles, Paris, Laffont, 1982 et dir. Michel Cazenave, Encyclopédie des symboles, Paris, Le Livre de Poche, 1992. 
ment plus généraux, plus universellement représentatifs, car le type du paysan se retrouve à peu près pareil à travers le monde entier [...]. [J']aurais voulu que le paysan fût pour moi, toutes proportions gardées, un peu ce que sont les rois dans Corneille et Racine : $\mathrm{j}$ 'entends un homme [...] étroitement soumis aux grandes contingences cycliques d'un climat et des saisons. ${ }^{(58)}$

Le sacrifice du bouc renvoie enfin, du bélier d'Abraham au bouc émissaire dans le Lévitique, au rôle purificateur de la mort rituelle qui permet, à l'intérieur de la communauté, de concentrer les manifestations de violence sur un seul individu et de régénérer, renforcer l'ordre social. Ainsi la chute finale de Jean-Luc le persécuté, celui sur qui tous les malheurs s'accumulent, en ferait un Christ bon pasteur, variante de l'agneau de Dieu, qui s'offre à la mort pour le salut des pécheurs. Ce drame de l'homme trahi peut être lu désormais comme une représentation universelle de la victime expiatoire : le chant du bouc sacrifié ${ }^{(59)}$.

\section{Ouvertures}

Seule l'adoption d'un angle d'analyse ethnocritique, cherchant à lire la fiction littéraire "dans sa réappropriation polyphonique des données du culturel » ${ }^{(60)}$, permet de mettre à jour les fondations traditionnelles sur lesquelles se met en place l'intrigue ${ }^{(61)}$. Nous sommes dans un monde perturbé où Jean-Luc, renié par sa mère, prend la place de la femme et bouleverse jusqu'à l'absurdité les règles élémentaires de la filiation. De même qu'en niant la disparition de son enfant, il remet en question les frontières entre morts et vivants.

On l'a vu, le modèle du village montagnard est le décor idéal pour le projet littéraire de Ramuz. Cependant, le travail de type ethnographique effectué par l'auteur ne le conduit pas à donner une image idéalisée de cet univers en vase clos où règne une culture essentiellement orale qui, selon Claude Lévi-Strauss, serait le garant d'une authenticité dans la communication et les rapports sociaux ${ }^{(62)}$. Le village où se déroule le destin tragique de Jean-Luc, incompris et rejeté par sa communauté jusqu'au sacrifice, n'a rien du cadre pastoral dont on serait nostalgique.

L'utilisation et la transformation des traits de folklore local traditionnel, où les rituels prennent dans la vie quotidienne « le relais du mythe » ${ }^{(63)}$, permettent au poète, dans un nouveau geste créateur, d'atteindre à la permanence du récit universel. N'est-ce pas ce dessein d'universalité qui se manifeste lors de la ré-

(58) C. F. Ramuz, Une province qui n'en est pas une, voir Essais, 3, édités par A. Rochat, OC, XVII, 2011, p. 252.

(59) A propos de la figure du Christ en bouc émissaire, voir René Girard,Le Bouc émissaire, Paris, Grasset, 1982.

(60) Présentation de l'enseignement de Jean-Marie Privat et Marie Scarpa sur le site internet consacré à l'ethnocritique : www.ethnocritique.com/page_4.html

(61) $\mathrm{Si}$, dans un premier temps, les principales études ethnocritiques ont pris pour objet les œuvres des grands romanciers du XIX ${ }^{\mathrm{e}}$ siècle (Flaubert, Zola), les outils proposés par cette orientation de recherche se révèlent pertinents à l'épreuve d'autres corpus, hors du cadre et de l'esthétique du récit réaliste. Les conclusions de cette présente étude en témoignent.

(62) Claude Lévi-Strauss, Anthropologie structurale, Paris, Plon Pocket, [1958], 2008, pp. 425428.

(63) Henri Rey-Flaud, Charivari, les rituels fondamentaux de la sexualité, Paris, Payot, 1985, p. 17. 
écriture du roman, en 1919 ? Les modifications introduites semblent répondre à cette volonté d'atteindre au général par le particulier qui, pour Ramuz, ne «peut être [...] qu'un point de départ » ${ }^{(64)}$. En comparant la fin de la version originale (1908) avec la deuxième (1919), on remarque que l'écrivain supprime le paragraphe qui achève le récit :

Et maintenant on parle encore de Jean-Luc là-haut et de sa folie. Même il en existe qui croient que l'enfant était vraiment revenu.

Et quelquefois, quand le soir tombe, s'étant signés ils disent bas : « La preuve, c'est que là où on l'a trouvé, il y avait deux mares de sang. " ${ }^{(65)}$

Ces quelques lignes disparaissent en effet, et les versions suivantes, à quelques modifications de détails près, s'achèvent ainsi :

On lui avait noué la tête dans un linge ; ils dirent : «Elle s'est fendue comme une noix ; la cervelle avait sauté dehors. »

Il y eut des portes ouvertes, des lanternes sorties, des voix ; Jean-Luc fut lourd à porter jusqu'au lit, où il demeura gisant.

Et il était grand sur le lit, il était tellement grand. ${ }^{(66)}$

En éliminant la première phrase, Ramuz épure son texte de toute possible référence spatiale ou temporelle : nous ne lisons plus désormais une histoire dont on parlerait « encore » « là-haut». Dans un même mouvement, il renonce également à la tension fantastique, créée par cette étrange double tache de sang, et s'éloigne définitivement de la légende montagnarde, appartenant à l'histoire orale d'un lieu.

Ainsi le créateur, qui veut faire du roman un poème ${ }^{(67)}$, n'est plus seulement lector, celui « qui commente le discours établi », mais auctor, celui « qui produit du discours nouveau » ${ }^{(68)}$. Ce que Ramuz lui-même dit en 1913 en affirmant que la poésie, dans un mouvement similaire à celui du mythe donnant à l'objet une dimension symbolique, " confère une sorte de divinité aux objets dont elle s'occupe, sans cesser d'y voir des objets ; elle est réaliste et symboliste tout à la fois, comme il faut être ; elle ne s'intéresse qu'à l'immédiat, mais par l'atmosphère dont elle l'entoure elle semble le transfigurer. » ${ }^{(69)}$

(64) C. F. Ramuz, Raison d'être, op. cit., p. 35.

(65) Jean-Luc persécuté, op. cit., p. 481.

(66) C. F. Ramuz, Romans, I, dir. Doris Jakubec, Paris, Pléiade, Gallimard, 2005, p. 382. La citation donne la dernière version de Jean-Luc persécuté, parue dans les Euvres complètes de 1940 (Lausanne, Mermod, vol. 4).

(67) Voir ci-dessus, note 4.

(68) Pierre Bourdieu, op. cit., p. 132.

(69) C. F. Ramuz, « Mythes », in Articles et chroniques, 2, OC, XII, 2008, p. 82. 